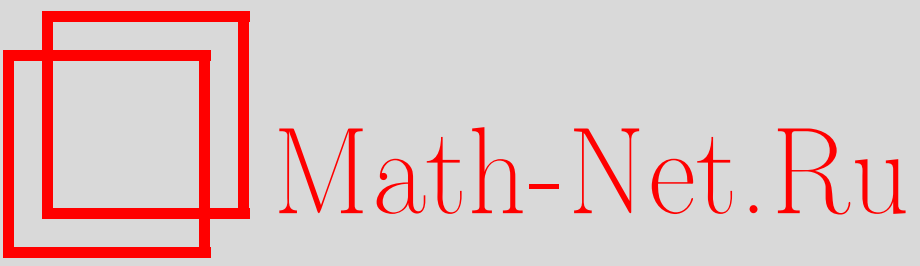

В. В. Жаринов, О когомологиях алгебры Пуассона, ТМФ, 2003, том 136, номер 2, 179-196

DOI: https://doi.org/10.4213/tmf228

Использование Общероссийского математического портала Math-Net.Ru подразумевает, что вы прочитали и согласны с пользовательским соглашением

http://www.mathnet.ru/rus/agreement

Параметры загрузки:

IP : 54.166 .219 .16

26 апреля 2023 г., 04:17:50

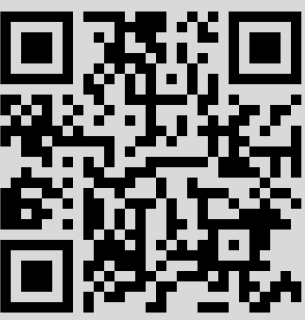


ТЕОРЕТИЧЕСКАЯ

И МАТЕМАТИЧЕСКАЯ

ФИЗИКА

Том 136, № 2

август, 2003

(C) 2003 г.

В.В. Жаринов*

\section{О КОГОМОЛОГИЯХ АЛГЕБРЫ ПУАССОНА}

Предложена техника вычислений когомологий алгебры Пуассона, использующая преобразование Лапласа обобщенных функций с компактным носителем. Найдены когомологии низших порядков этой алгебры с коэффициентами в двух естественных представлениях: тривиальном и присоединенном.

Ключевые слова: алгебры Ли, алгебры Пуассона, когомологии.

Когомологические методы глубоко проникли в современную математическую физику (см., например, [1], [2]) и даже привели к понятию когомологическая физика (см., например, [3]). Со своей стороны, скобки Пуассона и порождаемая ими одноименная алгебра Ли лежат в основе всех исследований динамики как классических, так и квантовых процессов, а изучение их когомологических свойств - активно развивающаяся область (см., например, [4]).

В настояшей работе изучаются когомологии алгебры Пуассона с коэффициентами в тривиальном и присоединенном представлениях. Используется техника вычислений, предложенная в работе [5] и основанная на преобразовании Лапласа обобшенных функций с компактным носителем. В частности, вычислены когомологии низших порядков, имеюшие естественные алгебро-геометрические интерпретации.

\section{1. ОСНОВНЫЕ ОПРЕДЕЛЕНИЯ}

1.1. Алгебра Пуассона. Пусть $V$-вешественное $m$-мерное векторное пространство и $V^{*}$ - его дуальное. Пусть также задан невырожденный бивектор $\chi \in \wedge^{2} V$, так что определен обратный элемент - биковектор $\chi^{-1} \in \wedge^{2} V^{*}$ (в частности, $m=2 D$ четное). При естественных отождествлениях $\chi: V \rightarrow V^{*}, \chi^{-1}: V^{*} \rightarrow V$ имеем $\chi \circ \chi^{-1}=\mathrm{id}_{V}$, $\chi^{-1} \circ \chi=\mathrm{id}_{V^{*}}$, или в координатах $\chi=\left(\chi^{\alpha \beta}\right), \chi^{\alpha \beta}+\chi^{\beta \alpha}=0, \operatorname{det}\left(\chi^{\alpha \beta}\right) \neq 0, \chi^{-1}=$ $\left(\chi_{\alpha \beta}\right), \sum_{\gamma} \chi^{\alpha \gamma} \chi_{\gamma \beta}=\sum_{\gamma} \chi_{\beta \gamma} \chi^{\gamma \alpha}=\delta_{\beta}^{\alpha}$, причем здесь и всюду ниже $\alpha, \beta, \gamma \in \overline{1, m}=$ $\{1, \ldots, m\}$.

Пусть $\mathcal{E}(V)=\mathcal{C}^{\infty}(V)$ - пространство всех гладких комплексных функций на $V$ с естественной топологией равномерной сходимости на компактах вместе с производными

* Математический институт им. В. А. Стеклова РАН, Москва, Россия. E-mail: zharinov@mi.ras.ru 
всех порядков. Для каждой пары $u, v \in \mathcal{E}(V)$ определена скобка Пуассон $\{\{u, v\}=$ $(d u \wedge d v, \chi) \in \mathcal{E}(V)$, или в координатах

$$
\{u, v\}(x)=\sum_{\alpha, \beta}\left(\partial_{x^{\alpha}} u(x)\right)\left(\partial_{x^{\beta}} v(x)\right) \chi^{\alpha \beta}, \quad x=\left(x^{1}, \ldots, x^{m}\right) \in V .
$$

Скобки Пуассона обладают стандартными свойствами скобок Ли: они билинейны, кососимметричны и удовлетворяют тождеству Якоби. Таким образом, определена алгебра Пуассона $\mathcal{P}=(\mathcal{E}(V) ;\{\cdot, \cdot\})$, т.е. определены линейное топологическое пространство $\mathcal{E}(V)$ и скобки Пуассона $\{\cdot, \cdot\}$ в качестве скобок Ли. Конечно, пространство $\mathcal{E}(V)$ обладает также структурой ассоциативной алгебры относительно обычных поточечных операций, но здесь она играет второстепенную роль.

1.2. Когомологии. Необходимые сведения об алгебрах Ли, их представлениях и когомологиях можно найти в книгах [6]-[8] и в обзоре [9]. Напомним здесь, что представление алгебры Ли $\mathfrak{A}$ в линейном пространстве $\mathfrak{K}$ есть линейное отображение $\pi: \mathfrak{A} \rightarrow$ $\mathfrak{g l}(\mathfrak{K})$, причем выполнено коммутаторное правило $\pi([a, b])=[\pi(a), \pi(b)]$, где $[a, b]-$ скобка Ли элементов $a, b \in \mathfrak{A}$, а $[\pi(a), \pi(b)]=\pi(a) \circ \pi(b)-\pi(b) \circ \pi(a)$ - коммутатор линейных отображений $\pi(a), \pi(b): \mathfrak{K} \rightarrow \mathfrak{K}$. Далее, пусть $\wedge \mathfrak{A}=\bigoplus_{q \geqslant 0} \wedge^{q} \mathfrak{A}-$ внешняя алгебра линейного пространства $\mathfrak{A}$ и $\mathcal{C}(\mathfrak{A} ; \mathfrak{K})=\bigoplus_{q \geqslant 0} \mathcal{C}^{q}(\mathfrak{A} ; \mathfrak{K})$ - линейное пространство всех коцепей на $\mathfrak{A}$ с коэффициентами в $\mathfrak{K}$, где $\mathcal{C}^{q}(\mathfrak{A} ; \mathfrak{K})$ - линейные пространства всех линейных отображений из $\wedge$ q $\mathfrak{A}$ в $\mathfrak{K}$ (другими словами, $q$-линейных кососимметрических отображений из $\mathfrak{A}^{q}=\times{ }^{q} \mathfrak{A}$ в $\left.\mathfrak{K}\right), q=0,1, \ldots$ Если пространства $\mathfrak{A}$ и $\mathfrak{K}$ наделены топологиями (как это имеет место в нашем случае), то все операции предполагаются непрерывными. Каждая алгебра Ли $\mathfrak{A}$ имеет два естественных представления, а именно тривиальное и присоединенное. В первом случае $\mathfrak{K}=\mathbb{C}$ - поле комплексных чисел, $\pi(a)=0$ для всех $a \in \mathfrak{A}$, а во втором $\mathfrak{K}=\mathfrak{A}$ и $(\pi(a))(b)=[a, b]$ для всех $a, b \in \mathfrak{A}$.

Внешний дифференциал $d$ на $\mathcal{C}(\mathfrak{A} ; \mathfrak{K})$ задается правилом

$$
\begin{aligned}
(d \omega)\left(a_{0}, \ldots, a_{q}\right)= & \frac{1}{q+1}\left\{\sum_{0 \leqslant i \leqslant q}(-1)^{i} \pi\left(a_{i}\right)\left(\omega\left(a_{0}, \ldots, \check{a}_{i}, \ldots, a_{q}\right)\right)+\right. \\
& \left.+\sum_{0 \leqslant i<j \leqslant q}(-1)^{i+j} \omega\left(\left[a_{i}, a_{j}\right], a_{0}, \ldots, \check{a}_{i}, \ldots, \check{a}_{j}, \ldots, a_{q}\right)\right\}
\end{aligned}
$$

для всех $\omega \in \mathcal{C}^{q}(\mathfrak{A} ; \mathfrak{K})$ и $a_{0}, \ldots, a_{q} \in \mathfrak{A}$ (аргумент под знаком "“" опускается), так что $d: \mathcal{C}^{q}(\mathfrak{A} ; \mathfrak{K}) \rightarrow \mathcal{C}^{q+1}(\mathfrak{A} ; \mathfrak{K}), q=0,1, \ldots$ В силу тождества Якоби и коммутаторного правила композиция $d \circ d=0$, так что определен комплекс $\{\mathcal{C}(\mathfrak{A} ; \mathfrak{K}) ; d\}$ с когомологиями

$$
H(\{\mathcal{C}(\mathfrak{A} ; \mathfrak{K}) ; d\})=H(\mathfrak{A} ; \mathfrak{K})=\bigoplus_{q \geqslant 0} H^{q}(\mathfrak{A} ; \mathfrak{K})
$$

В нашем случае $\mathfrak{A}=\mathcal{P}$ - алгебра Пуассона, и мы рассмотрим оба естественных представления. 
1.3. Тривиальное представление. В этом случае $\mathcal{C}^{q}(\mathcal{P} ; \mathbb{C})$ - линейные пространства всех $q$-линейных кососимметрических непрерывных функционалов на $\mathcal{P}^{q}$, наделенные сильной топологией. В частности, $q$-коцепи суть обобщенные функции с компактными носителями (элементы из пространства $\mathcal{E}\left(V^{q}\right)^{*}$, дуального к пространству $\left.\mathcal{E}\left(V^{q}\right)\right)$, обладающие кососимметрией. Внешний дифференциал $d_{\mathbb{C}}$ на $\mathcal{C}(\mathcal{P} ; \mathbb{C})$ действует по правилу

$$
\left(d_{\mathbb{C}} \omega\right)\left(u_{0}, \ldots, u_{q}\right)=\frac{1}{q+1} \sum_{0 \leqslant i<j \leqslant q}(-1)^{i+j} \omega\left(\left\{u_{i}, u_{j}\right\}, u_{0}, \ldots, \check{u}_{i}, \ldots, \check{u}_{j}, \ldots, u_{q}\right)
$$

для всех $\omega \in \mathcal{C}^{q}(\mathcal{P} ; \mathbb{C}), u_{0}, \ldots, u_{q} \in \mathcal{P}, q=0,1, \ldots$

1.4. Присоединенное представление. В этом случае $\mathcal{C}^{q}(\mathcal{P} ; \mathcal{P})$ - линейные пространства всех $q$-линейных кососимметрических непрерывных отображений из $\mathcal{P}^{q}$ в $\mathcal{P}$, наделенные сильной топологией. В частности, $q$-коцепи суть $\mathcal{P}$-значные обобщенные функции с компактными носителями, обладающие кососимметрией. Внешний дифференциал $d_{\mathcal{P}}$ на $\mathcal{C}(\mathcal{P} ; \mathcal{P})$ действует по правилу

$$
\begin{aligned}
\left(d_{\mathcal{P}} \omega\right)\left(x ; u_{0}, \ldots, u_{q}\right)= & \frac{1}{q+1}\left\{\sum_{0 \leqslant i \leqslant q}(-1)^{i}\left\{u_{i}, \omega\left(u_{0}, \ldots, \check{u}_{i}, \ldots, u_{q}\right)\right\}(x)+\right. \\
& \left.+\sum_{0 \leqslant i<j \leqslant q}(-1)^{i+j} \omega\left(x ;\left\{u_{i}, u_{j}\right\}, u_{0}, \ldots, \check{u}_{i}, \ldots, \check{u}_{j}, \ldots, u_{q}\right)\right\}
\end{aligned}
$$

для всех $\omega \in \mathcal{C}^{q}(\mathcal{P} ; \mathcal{P}), x \in V, u_{0}, \ldots, u_{q} \in \mathcal{P}, q=0,1, \ldots$

1.5. Экспоненциальное преобразование. Пусть $\mathbb{V}=V+i V$ и $\mathbb{V}^{*}=V^{*}+i V^{*}$ комплексификации пространств $V$ и $V^{*}$, соответственно. Каждому $\xi \in \mathbb{V}^{*}$ поставим в соответствие функцию $e^{\xi} \in \mathcal{E}(V)$, где $e^{\xi}(x)=e^{(\xi, x)}$ для всех $x \in V,(\xi, x)$-естественное спаривание ковектора и вектора, в координатах $(\xi, x)=\sum_{\alpha} \xi_{\alpha} x^{\alpha}, \xi=\left(\xi_{1}, \ldots, \xi_{m}\right), x=$ $\left(x^{1}, \ldots, x^{m}\right)$. Очевидно, $\left\{e^{\xi}, e^{\eta}\right\}=\langle\xi, \eta\rangle e^{\xi+\eta}$ для всех $\xi, \eta \in \mathbb{V}^{*}$, где $\langle\xi, \eta\rangle=(\xi \wedge \eta, \chi)-$ естественное спаривание биковектора и бивектора, в координатах

$$
\langle\xi, \eta\rangle=\sum_{\alpha, \beta} \xi_{\alpha} \eta_{\beta} \chi^{\alpha \beta}
$$

Пусть $q=0,1, \ldots$. Обозначим через $\operatorname{Exp}\left(\left(\mathbb{V}^{*}\right)^{q}\right)$ пространство всех целых функций экспоненциального типа на $\left(\mathbb{V}^{*}\right)^{q} \simeq \mathbb{C}^{m q}$. Преобразование Лапласа $\omega \mapsto L[\omega]$ определено формулой

$$
L[\omega]\left(\xi_{1}, \ldots, \xi_{q}\right)=\omega\left(e^{\xi_{1}}, \ldots, e^{\xi_{q}}\right) \quad \forall \omega \in \mathcal{E}\left(V^{q}\right)^{*}, \quad \xi_{1}, \ldots, \xi_{q} \in \mathbb{V}^{*} .
$$

Линейное отображение $L: \mathcal{E}\left(V^{q}\right)^{*} \rightarrow \operatorname{Exp}\left(\left(\mathbb{V}^{*}\right)^{q}\right)$ инъективное, его образ $L\left[\mathcal{E}\left(V^{q}\right)^{*}\right]$ состоит из всех целых функций на $\left(\mathbb{V}^{*}\right)^{q}$, растущих не быстрее линейной экспоненты в вещественных направлениях и не быстрее степени в мнимых направлениях. Линейное пространство $L\left[\mathcal{E}\left(V^{q}\right)^{*}\right]$ наделяется топологией, индуцированной из $\mathcal{E}\left(V^{q}\right)^{*}$, так что определен изоморфизм линейных топологических пространств $L: \mathcal{E}\left(V^{q}\right)^{*} \simeq L\left[\mathcal{E}\left(V^{q}\right)^{*}\right]($ см., например, [10]). 
По построению пространство $\mathcal{C}^{q}(\mathcal{P} ; \mathbb{C})$ есть замкнутое подпространство в $\mathcal{E}\left(V^{q}\right)^{*}$. Наделяя образ $\Phi^{q}=L\left[\mathcal{C}^{q}(\mathcal{P} ; \mathbb{C})\right]$ топологией, индуцированной из $L\left[\mathcal{E}\left(V^{q}\right)^{*}\right]$, получаем изоморфизм линейных топологических пространств $L: \mathcal{C}^{q}(\mathcal{P} ; \mathbb{C}) \simeq \Phi^{q}$. Пространство $\Phi^{q}$ состоит из всех целых функций $\phi\left(\xi_{1}, \ldots, \xi_{q}\right)$, растущих не быстрее линейной экспоненты в вещественных направлениях и не быстрее степени в мнимых направлениях и кососимметрических по аргументам $\xi_{1}, \ldots, \xi_{q} \in \mathbb{V}^{*}$. Оно наделено естественной топологией, конкретный вид которой для нас здесь не важен, мы лишш будем следить, явно об этом не упоминая, чтобы все вводимые операции были непрерывными. Положив $\Phi=\bigoplus_{q \geqslant 0} \Phi^{q}$, получим изоморфизм $L: \mathcal{C}(\mathcal{P} ; \mathbb{C}) \simeq \Phi$. Внешний дифференциал $d_{\mathbb{C}}$ на $\mathcal{C}(\mathcal{P} ; \mathbb{C})$ индуцирует одноименный внешний дифференциал $d_{\mathbb{C}}$ на $\Phi$, действуюший по правилу

$$
\left(d_{\mathbb{C}} \phi\right)\left(\xi_{0}, \ldots, \xi_{q}\right)=\frac{1}{q+1} \sum_{0 \leqslant i<j \leqslant q}(-1)^{i+j}\left\langle\xi_{i}, \xi_{j}\right\rangle \phi\left(\xi_{i}+\xi_{j}, \xi_{0}, \ldots, \check{\xi}_{i}, \ldots, \check{\xi}_{j}, \ldots, \xi_{q}\right)
$$

для всех $\phi \in \Phi^{q}, \xi_{0}, \ldots, \xi_{q} \in \mathbb{V}^{*}, q=0,1, \ldots$

Итак, справедливо

ПРЕДЛОЖЕНИЕ 1. Определен комплекс $\left\{\Phi ; d_{\mathbb{C}}\right\}$, причем имеет место изоморфизм комплексов

$$
L:\left\{\mathcal{C}(\mathcal{P} ; \mathbb{C}) ; d_{\mathbb{C}}\right\} \simeq\left\{\Phi ; d_{\mathbb{C}}\right\}
$$

Далее, для каждого $q=0,1, \ldots$ линейное пространство $\mathcal{C}^{q}(\mathcal{P} ; \mathcal{P})=\mathcal{P} \widehat{\otimes} \mathcal{C} q(\mathcal{P} ; \mathbb{C})$, где символ $\widehat{\otimes}$ означает замыкание тензорного произведения в подходяшей топологии. Отождествление $L \equiv \operatorname{id}_{\mathcal{P}} \otimes L$ распространяет преобразование Лапласа на $\mathcal{C}^{q}(\mathcal{P} ; \mathcal{P})$ и приводит к изоморфизму $L: \mathcal{C}^{q}(\mathcal{P} ; \mathcal{P}) \simeq \mathcal{F}^{q}$, где $\mathcal{F}^{q}=\mathcal{P} \widehat{\otimes} \Phi^{q}$. Введем "подкрученное" преобразование $\Lambda: \mathcal{C}^{q}(\mathcal{P} ; \mathcal{P}) \simeq \mathcal{F}^{q}$ правилом

$$
\Lambda[\omega]\left(x ; \xi_{1}, \ldots, \xi_{q}\right)=\exp \left(-\sum_{1 \leqslant i \leqslant q}\left(\xi_{i}, x\right)\right) L[\omega]\left(x ; \xi_{1}, \ldots, \xi_{q}\right)
$$

для всех $\omega \in \mathcal{C}^{q}(\mathcal{P} ; \mathcal{P}), x \in V, \xi_{1}, \ldots, \xi_{q} \in \mathbb{V}^{*}$. Таким образом, определен изоморфизм $\Lambda: \mathcal{C}(\mathcal{P} ; \mathcal{P}) \simeq \mathcal{F}$, где $\mathcal{F}=\bigoplus_{q \geqslant 0} \mathcal{F}^{q}$. Внешний дифференциал $d_{\mathcal{P}}$ на $\mathcal{C}(\mathcal{P} ; \mathcal{P})$ индуцирует внешний дифференциал $d_{\mathcal{P}}$ на $\mathcal{F}$, действующий по правилу

$$
\begin{aligned}
\left(d_{\mathcal{P}} f\right)\left(x ; \xi_{0}, \ldots, \xi_{q}\right)= & \frac{1}{q+1}\left\{\sum_{0 \leqslant i \leqslant q}(-1)^{i}\left\langle\xi_{i}, \sum_{j \neq i} \xi_{j}+\partial_{x}\right\rangle f\left(x ; \xi_{0}, \ldots, \check{\xi}_{i}, \ldots, \xi_{q}\right)+\right. \\
& \left.+\sum_{0 \leqslant i<j \leqslant q}(-1)^{i+j}\left\langle\xi_{i}, \xi_{j}\right\rangle f\left(x ; \xi_{i}+\xi_{j}, \xi_{0}, \ldots, \check{\xi}_{i}, \ldots, \check{\xi}_{j}, \ldots, \xi_{q}\right)\right\}
\end{aligned}
$$

для всех $f \in \mathcal{F}^{q}, x \in V, \xi_{0}, \ldots, \xi_{q} \in \mathbb{V}^{*}, q=0,1, \ldots$

Итак, справедливо

ПРЕДЛОЖЕНИЕ 2. Определен комплекс $\left\{\mathcal{F} ; d_{\mathcal{P}}\right\}$, причем имеет место изоморфизм комплексов

$$
\Lambda:\left\{\mathcal{C}(\mathcal{P} ; \mathcal{P}) ; d_{\mathcal{P}}\right\} \simeq\left\{\mathcal{F} ; d_{\mathcal{P}}\right\}
$$




\section{2. ТРИВИАЛЬНОЕ ПРЕДСТАВЛЕНИЕ}

В силу изоморфизма (1.1) достаточно изучить когомологии комплекса $\left\{\Phi ; d_{\mathbb{C}}\right\}$.

2.1. Случай $q=0$. По построению $\Phi^{0}=\mathbb{C}$, причем $\left.d_{\mathbb{C}}\right|_{\Phi^{0}}=0$, так что

$$
H^{0}(\mathcal{P} ; \mathbb{C}) \simeq H^{0}\left(\left\{\Phi ; d_{\mathbb{C}}\right\}\right)=\mathbb{C} .
$$

2.2. Случай $q=1$. Пусть $\phi \in \Phi^{1}$, тогда

$$
\left(d_{\mathbb{C}} \phi\right)(\xi, \eta)=-\frac{1}{2}\langle\xi, \eta\rangle \phi(\xi+\eta) \quad \forall \xi, \eta \in \mathbb{V}^{*} .
$$

Очевидно, $d_{\mathbb{C}} \phi=0$ тогда и только тогда, когда $\phi=0$. Учитывая, что $\left.d_{\mathbb{C}}\right|_{\Phi^{0}}=0$, получаем

$$
H^{1}(\mathcal{P} ; \mathbb{C}) \simeq H^{1}\left(\left\{\Phi ; d_{\mathbb{C}}\right\}\right)=0 .
$$

2.3. Случай $q=2$. Пусть $\phi \in \Phi^{2}$. Условие $d_{\mathbb{C}} \phi=0$ имеет вид

$$
\langle\xi, \eta\rangle \phi(\xi+\eta, \zeta)+\text { ц.п. }=0
$$

для всех $\xi, \eta, \zeta \in \mathbb{V}^{*}$, где сокращение "ц.п." означает циклическую перестановку по аргументам $\xi, \eta, \zeta \in \mathbb{V}^{*}$. В частности, $\langle\xi, \eta\rangle \phi(\xi+\eta, \zeta)=0$ при всех $\langle\xi, \zeta\rangle=\langle\eta, \zeta\rangle=0$. Следовательно, и $\phi(\xi, \zeta)=0$ при всех $\langle\xi, \zeta\rangle=0$, откуда $\phi(\xi, \zeta)=\langle\xi, \zeta\rangle \psi(\xi, \zeta)$, где $\psi(\xi, \zeta)-$ целая функция, с оценками роста того же типа, что и у функции $\phi(\xi, \zeta)$, причем $\psi(\xi, \zeta)=$ $\psi(\zeta, \xi)$ для всех $\xi, \zeta \in \mathbb{V}^{*}$. Подставляя это представление в (2.1), получаем

$$
\langle\xi, \eta\rangle\langle\xi+\eta, \zeta\rangle \psi(\xi+\eta, \zeta)+\text { ц.п. }=0,
$$

иначе

$$
\langle\xi, \eta\rangle\langle\xi, \zeta\rangle \Delta \psi(\xi, \eta, \zeta)+\text { ц.п. }=0,
$$

где $\Delta \psi(\xi, \eta, \zeta)=\psi(\xi+\eta, \zeta)-\psi(\xi+\zeta, \eta)$, для всех $\xi, \eta, \zeta \in \mathbb{V}^{*}$. Легко проверяется тождество

$$
\Delta \psi(\xi, \eta, \zeta)+\text { ц.п. }=0
$$

для всех $\psi(\xi, \eta)$ и $\xi, \eta, \zeta \in \mathbb{V}^{*}$, так что $\Delta \psi(\zeta, \xi, \eta)=-\Delta \psi(\xi, \eta, \zeta)-\Delta \psi(\eta, \zeta, \xi)$. Подставляя последнее равенство в $(2.2)$, получаем

$$
(\langle\xi, \eta\rangle\langle\xi, \zeta\rangle-\langle\zeta, \xi\rangle\langle\zeta, \eta\rangle) \Delta \psi(\xi, \eta, \zeta)+(\langle\eta, \xi\rangle\langle\eta, \zeta\rangle-\langle\zeta, \xi\rangle\langle\zeta, \eta\rangle) \Delta \psi(\eta, \zeta, \xi)=0,
$$

и, следовательно,

$$
\begin{aligned}
\langle\xi, \zeta\rangle\langle\xi+\zeta, \eta\rangle \Delta \psi(\xi, \eta, \zeta) & =\langle\zeta, \eta\rangle\langle\eta+\zeta, \xi\rangle \Delta \psi(\eta, \zeta, \xi)= \\
& =\langle\xi, \zeta\rangle\langle\zeta, \eta\rangle\langle\xi+\zeta, \eta\rangle\langle\eta+\zeta, \xi\rangle g(\xi, \eta, \zeta),
\end{aligned}
$$

где $g(\xi, \eta, \zeta)$ - некоторая целая функция. Отсюда

$$
\Delta \psi(\xi, \eta, \zeta)=\psi(\xi+\eta, \zeta)-\psi(\xi+\zeta, \eta)=\langle\zeta, \eta\rangle\langle\eta+\zeta, \xi\rangle g(\xi, \eta, \zeta) .
$$

Полагая в этом представлении $\eta=0$, получаем

$$
\psi(\xi, \zeta)=-\frac{1}{2} \rho(\xi+\zeta) \quad \forall \xi, \zeta \in \mathbb{V}^{*},
$$

где $\rho(\xi)=-2 \psi(\xi, 0) \in \Phi^{1}$. Другими словами, $\phi=d_{\mathbb{C}} \rho$ и, значит,

$$
H^{2}(\mathcal{P} ; \mathbb{C}) \simeq H^{2}\left(\left\{\Phi ; d_{\mathbb{C}}\right\}\right)=0 .
$$


2.4. Обсуждение. Равенство $H^{0}(\mathcal{P} ; \mathbb{C})=\mathbb{C}$ специальной информации об алгебре Пуассона не несет, поскольку аналогичное равенство справедливо для всех алгебр Ли и отражает лиш билинейность скобки Ли. Равенство $H^{1}(\mathcal{P} ; \mathbb{C})=0$ эквивалентно известному соотношению $\{\mathcal{P} ; \mathcal{P}\}=\mathcal{P}$ и отражает полноту скобок Пуассона. Наконец, равенство $H^{2}(\mathcal{P} ; \mathbb{C})=0$ означает, что с точки зрения алгебр Ли алгебра Пуассона не имеет нетривиальных одномерных чентральных расширений.

\section{3. ПРИСОЕДИНЕННОЕ ПРЕДСТАВЛЕНИЕ}

В силу изоморфизма $\Lambda:\left\{\mathcal{C}(\mathcal{P} ; \mathcal{P}) ; d_{\mathcal{P}}\right\} \simeq\left\{\mathcal{F} ; d_{\mathcal{P}}\right\}$ достаточно изучить когомологии комплекса $\left\{\mathcal{F} ; d_{\mathcal{P}}\right\}$. Заметим, что отождествление $\Phi \equiv 1_{\mathcal{P}} \otimes \Phi$, где $1_{\mathcal{P}} \in \mathcal{P}, 1_{\mathcal{P}}(x) \equiv 1$, определяет вложение $\Phi \in \mathcal{F}$. С другой стороны, всякое линейное отображение $A: \Phi \rightarrow \Phi$ распространяется на $\mathcal{F}$ отождествлением $A \equiv \mathrm{id}_{\mathcal{P}} \otimes A: \mathcal{F} \rightarrow \mathcal{F}$.

3.1. Внешний дифференциал. Прежде всего разобьем внешний дифференциал $d_{\mathcal{P}}: \mathcal{F} \rightarrow \mathcal{F}$ на два естественных слагаемых: $d_{\mathcal{P}}=\delta+\nabla$. Отображение $\delta: \mathcal{F} \rightarrow \mathcal{F}$ определено правилом

$$
(\delta f)\left(x ; \xi_{0}, \ldots, \xi_{q}\right)=\frac{1}{q+1} \sum_{0 \leqslant i \leqslant q}(-1)^{i}\left\langle\xi_{i}, \partial_{x}\right\rangle f\left(x ; \xi_{0}, \ldots, \check{\xi}_{i}, \ldots, \xi_{q}\right)
$$

для всех $f \in \mathcal{F}^{q}, x \in V, \xi_{0}, \ldots, \xi_{q} \in \mathbb{V}^{*}, q=0,1, \ldots$ В свою очередь, отображение $\nabla: \Phi \rightarrow \Phi$ действует по формуле

$(\nabla \phi)\left(\xi_{0}, \ldots, \xi_{q}\right)=$

$$
=\frac{1}{q+1} \sum_{0 \leqslant i<j \leqslant q}(-1)^{i+j}\left\langle\xi_{i}, \xi_{j}\right\rangle\left(\nabla_{\xi_{i}} \xi_{j} \phi\right)\left(\xi_{0}, \ldots, \check{\xi}_{i}, \ldots, \check{\xi}_{j}, \ldots, \xi_{q}\right)
$$

для всех $\phi \in \Phi^{q}, \xi_{0}, \ldots, \xi_{q} \in \mathbb{V}^{*}, q=0,1, \ldots$, где

$$
\left(\nabla_{\xi \eta} \phi\right)\left(\zeta_{1}, \ldots, \zeta_{q-1}\right)=\phi\left(\xi+\eta, \zeta_{1}, \ldots, \zeta_{q-1}\right)-\phi\left(\xi, \zeta_{1}, \ldots, \zeta_{q-1}\right)-\phi\left(\eta, \zeta_{1}, \ldots, \zeta_{q-1}\right)
$$

для всех $\xi, \eta, \zeta_{1}, \ldots, \zeta_{q-1} \in \mathbb{V}^{*}$.

ПРЕДЛОЖЕНИЕ 3. Линейнъе отображсения $\delta, \nabla: \mathcal{F} \rightarrow \mathcal{F}$ обладают свойствами

$$
\delta \circ \delta=0, \quad \delta \circ \nabla+\nabla \circ \delta=0, \quad \nabla \circ \nabla=0 .
$$

В частности, $\delta$ и $\nabla$-внешние дифференциаль на $\mathcal{F}$.

ДОКАЗАТЕЛЬСТВО проводится прямыми вычислениями. 
3.2. Вспомогательные операции. Линейное отображение $\iota: \Phi \rightarrow \Phi, \Phi^{q} \ni \phi \mapsto$ $\iota \phi \in \Phi^{q-1}$, зададим правилом

$$
(\iota \phi)\left(\xi_{1}, \ldots, \xi_{q-1}\right)=q \phi\left(0, \xi_{1}, \ldots, \xi_{q-1}\right)
$$

для всех $\xi_{1}, \ldots, \xi_{q-1} \in \mathbb{V}^{*}, q=0,1, \ldots$ Легко проверить, что

$$
d_{\mathcal{P}} \circ \iota+\iota \circ d_{\mathcal{P}}=0
$$

Далее, каждой паре $f \in \mathcal{F}^{p}, g \in \mathcal{F}^{q}$ поставим в соответствие внешнее произведение $f \wedge g \in \mathcal{F}^{p+q}$, определяемое равенством

$$
\begin{aligned}
& (f \wedge g)\left(x ; \xi_{1}, \ldots, \xi_{p+q}\right)= \\
& \quad=\frac{1}{(p+q) !} \sum_{\sigma \in \Sigma_{p+q}} \operatorname{sign} \sigma f\left(x ; \xi_{\sigma(1)}, \ldots, \xi_{\sigma(p)}\right) g\left(x ; \xi_{\sigma(p+1)}, \ldots, \xi_{\sigma(p+q)}\right)
\end{aligned}
$$

для всех $x \in V, \xi_{1}, \ldots, \xi_{p+q} \in \mathbb{V}^{*}$, где $\operatorname{sign} \sigma$ - знак перестановки $\sigma$ индексов $\{1, \ldots$ $\ldots, p+q\}$. В частности, для $p=0,1,2$ имеем соответственно

$$
\begin{aligned}
(f \wedge g)\left(x ; \xi_{1}, \ldots, \xi_{q}\right) & =f(x) g\left(x ; \xi_{1}, \ldots, \xi_{q}\right), \\
(f \wedge g)\left(x ; \xi_{0}, \ldots, \xi_{q}\right) & =\frac{1}{q+1} \sum_{0 \leqslant i \leqslant q}(-1)^{i} f\left(x ; \xi_{i}\right) g\left(x ; \xi_{0}, \ldots, \check{\xi}_{i}, \ldots, \xi_{q}\right), \\
(f \wedge g)\left(x ; \xi_{0}, \ldots, \xi_{q+1}\right)= & \frac{2}{(q+1)(q+2)} \sum_{0 \leqslant i<j \leqslant q+1}(-1)^{i+j-1} f\left(x ; \xi_{i}, \xi_{j}\right) \times \\
& \times g\left(x ; \xi_{0}, \ldots, \check{\xi}_{i}, \ldots, \check{\xi}_{j}, \ldots, \xi_{q+1}\right) .
\end{aligned}
$$

Билинейная операция $\wedge$ задает в линейном пространстве $\mathcal{F}$ структуру внешней алгебры, а линейные отображения $\iota, \delta, \nabla$ суть внешние дифференцирования этой алгебры. Именно,

$$
\begin{aligned}
\iota(f \wedge g) & =(\iota f) \wedge g+(-1)^{p} f \wedge(\iota g), \\
\delta(f \wedge g) & =(\delta f) \wedge g+(-1)^{p} f \wedge(\delta g), \\
\nabla(f \wedge g) & =(\nabla f) \wedge g+(-1)^{p} f \wedge(\nabla g)
\end{aligned}
$$

для всех $f \in \mathcal{F}^{p}, g \in \mathcal{F}^{q}$.

Каждый элемент $u \in \mathcal{F}^{p}$ задает линейное отображение $\hat{u}: \mathcal{F} \rightarrow \mathcal{F}, \mathcal{F}^{q} \ni f \mapsto \hat{u} f=$ $u \wedge f \in \mathcal{F}^{q+p}$. В частности, для $e \in \mathcal{F}^{1}, e(x ; \xi)=1-(\xi, x) / 2$, где $x \in V, \xi \in \mathbb{V}^{*},(\xi, x)=$ $\sum_{\alpha} \xi_{\alpha} x^{\alpha}$, имеем

$$
\begin{gathered}
\iota \circ \iota=0, \quad \iota \circ \hat{e}+\hat{e} \circ \iota=\mathrm{id}, \quad \hat{e} \circ \hat{e}=0, \\
d_{\mathcal{P}} \circ \hat{e}+\hat{e} \circ d_{\mathcal{P}}=0,
\end{gathered}
$$

поскольку $\iota е=1 \in \mathcal{F}^{0}(1(x) \equiv 1)$ и $d_{\mathcal{P}} e=0$. 
3.3. Расщепление комплекса $\left\{\mathcal{F} ; d_{\mathcal{P}}\right\}$. Положим $\mathrm{pr}_{\iota}=\iota \circ \hat{e}$ и $\mathrm{pr}_{e}=\hat{e} \circ \iota$. $\mathrm{B}$ силу равенств (3.6) имеем $\mathrm{pr}_{\iota} \circ \mathrm{pr}_{\iota}=\mathrm{pr}_{\iota}, \mathrm{pr}_{e} \circ \mathrm{pr}_{e}=\mathrm{pr}_{e}$, причем $\mathrm{pr}_{\iota}+\mathrm{pr}_{e}=\mathrm{id}$. Другими словами, проекторы $\mathrm{pr}_{\iota}$ и $\mathrm{pr}_{e}$ разлагают пространство $\mathcal{F}$ в прямую сумму двух подпространств. Первое подпространство $\mathcal{F}_{\iota}=\operatorname{pr}_{\iota} \mathcal{F}=\operatorname{ker} \iota=\{f \in \mathcal{F}: \iota f=0\}$, а второе $\mathcal{F}_{e}=\operatorname{pr}_{e} \mathcal{F}=\operatorname{ker} \hat{e}=\{f \in \mathcal{F}: \hat{e} f=0\}$ в силу тех же равенств $(3.6)$. Итак, $\mathcal{F}=\mathcal{F}_{\iota} \oplus \mathcal{F}_{e}$, причем в силу равенств (3.4) и (3.7) имеем

$$
d_{\mathcal{P}}: \mathcal{F}_{\iota} \rightarrow \mathcal{F}_{\iota}, \quad d_{\mathcal{P}}: \mathcal{F}_{e} \rightarrow \mathcal{F}_{e}
$$

так что определены подкомплексы $\left\{\mathcal{F}_{\iota} ; d_{\mathcal{P}}\right\}$ и $\left\{\mathcal{F}_{e} ; d_{\mathcal{P}}\right\}$. Из приведенных рассуждений следует, что справедливо

ПРЕДЛОЖЕНИЕ 4. Имеет место расщепление

$$
H^{q}\left(\left\{\mathcal{F} ; d_{\mathcal{P}}\right\}\right)=H^{q}\left(\left\{\mathcal{F}_{\iota} ; d_{\mathcal{P}}\right\}\right) \oplus H^{q}\left(\left\{\mathcal{F}_{e} ; d_{\mathcal{P}}\right\}\right), \quad q=0,1, \ldots
$$

Далее, из равенств (3.6) следует, что

$$
\hat{e}: \mathcal{F}_{\iota}^{q} \simeq \mathcal{F}_{e}^{q+1}, \quad \iota: \mathcal{F}_{e}^{q} \simeq \mathcal{F}_{\iota}^{q-1}, \quad q=0,1, \ldots
$$

причем эти изоморфизмы взаимно обратные, например $\mathcal{F}_{\iota}^{q}=\mathcal{F}_{\iota} \cap \mathcal{F}^{q}=\left\{f \in \mathcal{F}^{q}\right.$ : $\iota f=0\}$. Учитывая равенства (3.4) и (3.7), приходим к выводу, что справедливо

ПРЕДЛОЖЕНИЕ 5. Имеют место изоморфизмы

$$
H^{q}\left(\left\{\mathcal{F}_{\iota} ; d_{\mathcal{P}}\right\}\right) \simeq H^{q+1}\left(\left\{\mathcal{F}_{e} ; d_{\mathcal{P}}\right\}\right), \quad H^{q}\left(\left\{\mathcal{F}_{e} ; d_{\mathcal{P}}\right\}\right) \simeq H^{q-1}\left(\left\{\mathcal{F}_{\iota} ; d_{\mathcal{P}}\right\}\right),
$$

$q=0,1, \ldots$, индуцированные изоморфизмами $\hat{e} u \iota$.

Таким образом, достаточно знать когомологии хотя бы одного из комплексов $\left\{\mathcal{F}_{\iota} ; d_{\mathcal{P}}\right\},\left\{\mathcal{F}_{e} ; d_{\mathcal{P}}\right\}$. Например, в терминах первого комплекса имеем

$$
H^{q}\left(\left\{\mathcal{F} ; d_{\mathcal{P}}\right\}\right) \simeq H^{q}\left(\left\{\mathcal{F}_{\iota} ; d_{\mathcal{P}}\right\}\right) \times H^{q-1}\left(\left\{\mathcal{F}_{\iota} ; d_{\mathcal{P}}\right\}\right), \quad q=0,1, \ldots
$$

где $H^{-1}\left(\left\{\mathcal{F}_{\iota} ; d_{\mathcal{P}}\right\}\right)=0\left(\right.$ так же как и $\left.\mathcal{F}^{-1}\right)$.

3.4. Дополнительные операции. Выберем на $V$ и $\mathbb{V}^{*}$ согласованные системы координат. Обозначим через $\left\{\Omega ; d_{x}\right\}$ комплекс де Рама гладких дифференциальных форм на $V$, где $\Omega=\bigoplus_{p \geqslant 0} \Omega^{p}$,

$$
\Omega^{p}=\left\{\omega=\sum_{\alpha_{1}, \ldots, \alpha_{p}} \omega_{\alpha_{1} \ldots \alpha_{p}} d x^{\alpha_{1}} \wedge \cdots \wedge d x^{\alpha_{p}} ; \omega_{\alpha_{1} \ldots \alpha_{p}} \in \mathcal{E}(V)=\mathcal{P}\right\}
$$

а внешний дифференциал $d_{x}: \Omega \rightarrow \Omega, \Omega^{p} \ni \omega \mapsto d_{x} \omega \in \Omega^{p+1}$, действует по формуле

$$
d_{x} \omega=\sum_{\alpha_{0}, \ldots, \alpha_{p}} \partial_{x^{\left[\alpha_{0}\right.}} \omega_{\left.\alpha_{1}, \ldots, \alpha_{p}\right]} d x^{\alpha_{0}} \wedge \cdots \wedge d x^{\alpha_{p}}
$$


для всех $p=0,1, \ldots$, где [ $\ldots$ ] обозначает кососимметризацию по индексам, находящимся внутри квадратных скобок. В силу известной леммы Пуанкаре получаем, что когомологии $H^{0}\left(\left\{\Omega ; d_{x}\right\}\right)=\mathbb{C}, H^{p}\left(\left\{\Omega ; d_{x}\right\}\right)=0, p=1,2, \ldots$.

Введем биградуированное линейное пространство $\Omega \Phi=\bigoplus_{p, q \geqslant 0} \Omega^{p} \Phi^{q}$, где $\Omega^{p} \Phi^{q}=$ $\Omega^{p} \widehat{\otimes} \Phi^{q}$, в частности $\Omega^{0} \Phi^{q}=\mathcal{F}^{q}$. Всякое линейное отображение $X: \Omega \rightarrow \Omega$ распространяется на $\Omega \Phi$ отождествлением $X \equiv X \otimes \mathrm{id}_{\Phi}$, а всякое линейное отображение $A: \Phi \rightarrow \Phi$ распространяется на $\Omega \Phi$ отождествлением $A \equiv \mathrm{id} \Omega \otimes A$. Очевидно, отображения первого и второго типов всегда коммутируют. Введенное выше отображение $\delta: \mathcal{F} \rightarrow \mathcal{F}$ распространяется на $\Omega \Phi$ формулой

$$
\delta \omega=\sum_{\alpha_{1}, \ldots, \alpha_{p}} \delta \omega_{\alpha_{1} \ldots \alpha_{p}} d x^{\alpha_{1}} \wedge \cdots \wedge d x^{\alpha_{p}}
$$

для каждого $\omega \in \Omega^{p} \Phi^{q}$ (заметим, что коэффициенты $\omega_{\alpha_{1} \ldots \alpha_{p}} \in \mathcal{F}^{q}$ для всех $\alpha_{1}, \ldots, \alpha_{p} \in$ $\overline{1, m})$. Для каждого $\alpha \in \overline{1, m}$ определим линейное отображение $\kappa_{\alpha}: \Phi \rightarrow \Phi, \Phi^{q} \ni \phi \mapsto$ $\kappa_{\alpha} \phi \in \Phi^{q-1}$, правилом

$$
\left(\kappa_{\alpha} \phi\right)\left(\xi_{1}, \ldots, \xi_{q-1}\right)=\left.q \partial_{\xi^{\alpha}} \phi\left(\xi, \xi_{1}, \ldots, \xi_{q-1}\right)\right|_{\xi=0}
$$

для всех $\xi_{1}, \ldots, \xi_{q-1} \in \mathbb{V}^{*}$ (поясним, что $\xi^{\alpha}=\sum_{\beta} \xi_{\beta} \chi^{\beta \alpha}, \xi_{\alpha}=\sum_{\beta} \xi^{\beta} \chi_{\beta \alpha}$ ). Линейное отображение $\kappa: \Omega \Phi \rightarrow \Omega \Phi, \Omega^{p} \Phi^{q} \ni \omega \mapsto \kappa \omega \in \Omega^{p+1} \Phi^{q-1}$, зададим формулой

$$
\kappa \omega=\sum_{\alpha_{0}, \ldots, \alpha_{p}} \kappa_{\left[\alpha_{0}\right.} \omega_{\left.\alpha_{1} \ldots \alpha_{p}\right]} d x^{\alpha_{0}} \wedge \cdots \wedge d x^{\alpha_{p}} .
$$

Для каждого $\alpha \in \overline{1, m}$ определена координатная функция $\xi_{\alpha} \in \Phi^{1}$. Как показано в п. 3.2, ей отвечает линейное отображение $\widehat{\xi_{\alpha}}: \Phi \rightarrow \Phi, \Phi^{q} \ni \phi \mapsto \widehat{\xi_{\alpha}} \phi \in \Phi^{q+1}$, где

$$
\left(\widehat{\xi_{\alpha}} \phi\right)\left(\xi_{0}, \ldots, \xi_{q}\right)=\frac{1}{q+1} \sum_{0 \leqslant i \leqslant q}(-1)^{i} \xi_{i \alpha} \phi\left(\xi_{0}, \ldots, \check{\xi}_{i}, \ldots, \xi_{q}\right) .
$$

В частности, определено линейное отображение $\hat{\xi}: \Omega \Phi \rightarrow \Omega \Phi$, действуюшее по правилу $\Omega^{p} \Phi^{q} \ni \omega \mapsto \hat{\xi} \omega \in \Omega^{p+1} \Phi^{q+1}$, где

$$
\hat{\xi} \omega=\sum_{\alpha_{0}, \ldots, \alpha_{p}} \widehat{\xi_{\left[\alpha_{0}\right.}} \omega_{\left.\alpha_{1} \ldots \alpha_{p}\right]} d x^{\alpha_{0}} \wedge \cdots \wedge d x^{\alpha_{p}} .
$$

ПРЕДЛОЖЕНИЕ 6. Имеют место равенства

1) $\kappa_{\alpha} \circ \kappa_{\beta}+\kappa_{\beta} \circ \kappa_{\alpha}=0$ для всех $\alpha, \beta \in \overline{1, m}$;

2) $\kappa \circ \iota+\iota \circ \kappa=0$;

3) $\kappa \circ \delta+\delta \circ \kappa=d_{x}, \kappa \circ \nabla+\nabla \circ \kappa=\hat{\xi} \circ \iota$;

4) $\kappa \circ d_{x}+d_{x} \circ \kappa=0$;

5) $\widehat{\xi_{\alpha}} \circ \widehat{\xi_{\beta}}+\widehat{\xi_{\beta}} \circ \widehat{\xi_{\alpha}}=0$ для всех $\alpha, \beta \in \overline{1, m}$;

6) $\widehat{\xi_{\alpha}} \circ \iota+\iota \circ \widehat{\xi_{\alpha}}=0$ для всех $\alpha \in \overline{1, m}$;

7) $\widehat{\xi_{\alpha}} \circ \nabla+\nabla \circ \widehat{\xi_{\alpha}}=0$ для всех $\alpha \in \overline{1, m}$. 
ДОКАЗАТЕЛЬСТВО проводится прямыми вычислениями.

3.5. Изучение комплекса $\left\{\mathcal{F}_{\iota} ; d_{\mathcal{P}}\right\}$. По построению пространства $\Omega^{p} \Phi^{q}, p, q=$ $0,1, \ldots$, состоят из $p$-форм $\omega=\sum \omega_{\alpha_{1} \ldots \alpha_{p}} d x^{\alpha_{1}} \wedge \cdots \wedge d x^{\alpha_{p}}$ с коэффициентами $\omega_{\alpha_{1} \ldots \alpha_{p}} \in$ $\mathcal{F}^{q}$. Обозначим через $\Omega^{p} \Phi_{\iota}^{q}, \Omega_{\mathbb{C}}^{p} \Phi^{q}, \Omega_{\mathbb{C}}^{p} \Phi_{\iota}^{q}=\Omega_{\mathbb{C}}^{p} \Phi^{q} \cap \Omega^{p} \Phi_{\iota}^{q}$ их подпространства, состоящие из всех $p$-форм с коэффициентами $\omega_{\alpha_{1} \ldots \alpha_{p}} \in \mathcal{F}_{\iota}^{q}, \Phi^{q}, \Phi_{\iota}^{q}=\mathcal{F}_{\iota}^{q} \cap \Phi^{q}$, соответственно. В частности, $\Omega^{0} \Phi_{\iota}^{q}=\mathcal{F}_{\iota}^{q}, \Phi_{\iota}^{q}=\left\{\omega \in \mathcal{F}_{\iota}^{q}: d_{x} \omega=0\right\}$.

Положим $\kappa^{1}=\kappa, \kappa^{2}=\kappa \circ \kappa, \ldots$, так что $\kappa^{r}: \Omega^{p} \Phi^{q} \rightarrow \Omega^{p+r} \Phi^{q-r}$.

Лемма 1. Пусть $\omega \in \Omega^{p} \Phi_{\iota}^{q}, d_{\mathcal{P}} \omega=0$. Тогда

$$
d_{\mathcal{P}} \kappa^{r} \omega=r d_{x} \kappa^{r-1} \omega \quad \forall r=1,2, \ldots
$$

ДокАЗАТЕЛЬСТво. Для $r=1$ равенство $d_{\mathcal{P}} \kappa \omega=d_{x} \omega$ следует из п. 3 предложения 6. По индукции предположим, что $d_{\mathcal{P}} \kappa^{r} \omega=r d_{x} \kappa^{r-1} \omega$. Тогда для $r+1$ в силу тех же формул п. 3 с учетом п. 4 предложения 6 получим

$$
\begin{aligned}
d_{\mathcal{P}} \kappa^{r+1} \omega & =-\kappa d_{\mathcal{P}} \kappa^{r} \omega+d_{x} \kappa^{r} \omega=-r \kappa d_{x} \kappa^{r-1} \omega+d_{x} \kappa^{r} \omega= \\
& =r d_{x} \kappa^{r} \omega+d_{x} \kappa^{r} \omega=(r+1) d_{x} \kappa^{r} \omega,
\end{aligned}
$$

что и требовалось доказать.

Лемма 2. Пусть $\omega \in \Omega^{p} \Phi_{\iota}^{q}$. Равенство $d_{\mathcal{P}} \omega=0$ имеет место тогда и только тогда, когда справедливо представление

$$
\omega= \begin{cases}d_{\mathcal{P}} g+\phi, & p=0, \\ d_{\mathcal{P}} \rho+d_{x} \sigma, & p>0\end{cases}
$$

с некоторыми $g \in \mathcal{F}_{\iota}^{q-1}, \phi \in \Phi_{\iota}^{q}, \rho \in \Omega^{p} \Phi_{\iota}^{q-1}, \sigma \in \Omega^{p-1} \Phi_{\iota}^{q}$, где $\nabla \phi=0, d_{x} d_{\mathcal{P}} \sigma=0$.

ДокАЗАТЕЛЬСтво. Пусть $\omega \in \Omega^{p} \Phi_{\iota}^{q}$ и $d_{\mathcal{P}} \omega=0$. Положив в предыдущей лемме $r=$ $q+1$, получим $d_{x} \kappa^{q} \omega=0$, поскольку $\Omega^{p+q+1} \Phi^{-1}=0$. В силу леммы Пуанкаре отсюда следует, что $\kappa^{q} \omega=d_{x} \omega^{(0)}$ для некоторого $\omega^{(0)} \in \Omega^{p+q-1} \Phi_{\iota}^{0}$ (заметим, что $\kappa^{q} \omega \in$ $\left.\Omega^{p+q} \Phi_{\iota}^{0}\right)$. Полагая теперь в равенстве $(3.9) r=q$, получим $d_{\mathcal{P}} \kappa^{q} \omega=d_{\mathcal{P}} d_{x} \omega^{(0)}=q \times$ $d_{x} \kappa^{q-1} \omega$. Таким образом, $d_{x}\left(\kappa^{q-1} \omega-q^{-1} d_{\mathcal{P}} \omega^{(0)}\right)=0$, поскольку отображения $d_{x}$ и $d_{\mathcal{P}}$ коммутируют. Опять в силу леммы Пуанкаре имеем $\kappa^{q-1} \omega=d_{x} \omega^{(1)}+(1 / q) d_{\mathcal{P}} \omega^{(0)}$, где $\omega^{(1)} \in \Omega^{p+q-2} \Phi_{\iota}^{1}$. Равенство (3.9) теперь дает $d_{\mathcal{P}} \kappa^{q-1} \omega=d_{\mathcal{P}} d_{x} \omega^{(1)}=d_{x} \kappa^{q-2} \omega$. Продолжая в том же духе, на $q$-м шаге получим $\kappa^{1} \omega=d_{x} \omega^{(q-1)}+2^{-1} d_{\mathcal{P}} \omega^{(q-2)}$, где $\omega^{(q-1)} \in \Omega^{p} \Phi_{\iota}^{q-1}$. Равенство (3.9) на этом шаге дает $d_{\mathcal{P}} \kappa^{1} \omega=d_{\mathcal{P}} d_{x} \omega^{(q-1)}=d_{x} \omega$, откуда $d_{x}\left(\omega-d_{\mathcal{P}} \omega^{(q-1)}\right)=0$. Если $p=0$, то лемма Пуанкаре дает $\omega=d_{\mathcal{P}} \omega^{(q-1)}+\phi$, где $\omega^{(q-1)}=g \in \mathcal{F}_{\iota}^{q-1}, \phi \in \Phi_{\iota}^{q}$, причем $\nabla \phi=d_{\mathcal{P}} \phi=d_{\mathcal{P}}\left(\omega-d_{\mathcal{P}} g\right)=0$. Если же $p>0$, то лемма Пуанкаре дает $\omega=d_{\mathcal{P}} \omega^{(q-1)}+d_{x} \omega^{(q)}$, где $\omega^{(q-1)}=\rho \in \Omega^{p} \Phi_{\iota}^{q-1}, \omega^{(q)}=\sigma \in$ $\Omega^{p-1} \Phi_{\iota}^{q}$, причем $d_{x} d_{\mathcal{P}} \sigma=d_{\mathcal{P}}\left(\omega-d_{\mathcal{P}} \rho\right)=0$.

Обратное утверждение очевидно.

Доказанная лемма имеет следуюшее усиление. 
Лемма 3. Пусть $\omega \in \Omega^{p} \Phi_{\iota}^{q}$. Равенство $d_{\mathcal{P}} \omega=0$ имеет место тогда и только тогда, когда справедливо представление

$$
\omega=d_{\mathcal{P}} \rho+\tau, \quad \rho \in \Omega^{p} \Phi_{\iota}^{q-1}, \quad \tau \in \Omega_{\mathbb{C}}^{p} \Phi_{\iota}^{q}, \quad \nabla \tau=0 .
$$

ДОКАЗАТЕЛЬСТво. Пусть

$$
\omega=\sum \omega_{\alpha_{1} \ldots \alpha_{p}} d x^{\alpha_{1}} \wedge \cdots \wedge d x^{\alpha_{p}} \in \Omega^{p} \Phi_{\iota}^{q}, \quad d_{\mathcal{P}} \omega=0
$$

тогда каждый коэффициент $\omega_{\alpha_{1} \ldots \alpha_{p}} \in \mathcal{F}_{\iota}^{q}=\Omega^{0} \Phi_{\iota}^{q}$, причем $d_{\mathcal{P}} \omega_{\alpha_{1} \ldots \alpha_{p}}=0$. В силу предыдущей леммы $(p=0)$ имеем $\omega_{\alpha_{1} \ldots \alpha_{p}}=d_{\mathcal{P}} \rho_{\alpha_{1} \ldots \alpha_{p}}+\tau_{\alpha_{1} \ldots \alpha_{p}}$, где $\rho_{\alpha_{1} \ldots \alpha_{p}} \in \mathcal{F}_{\iota}^{q-1}$, $\tau_{\alpha_{1} \ldots \alpha_{p}} \in \Phi_{\iota}^{q}, \nabla \tau_{\alpha_{1} \ldots \alpha_{p}}=0$, причем без ограничения общности коэффициенты $\rho_{\alpha_{1} \ldots \alpha_{p}}$ и $\tau_{\alpha_{1} \ldots \alpha_{p}}$ можно считать кососимметрическими по индексам $\alpha_{1}, \ldots, \alpha_{p}$. Положив

$$
\rho=\sum \rho_{\alpha_{1} \ldots \alpha_{p}} d x^{\alpha_{1}} \wedge \cdots \wedge d x^{\alpha_{p}}, \quad \tau=\sum \tau_{\alpha_{1} \ldots \alpha_{p}} d x^{\alpha_{1}} \wedge \cdots \wedge d x^{\alpha_{p}},
$$

получим формы с требуемыми свойствами. Обратное утверждение очевидно.

ПРЕДЛОЖЕНИЕ 7. Пусть $\omega=\sum \omega_{\alpha_{1} \ldots \alpha_{p}} d x^{\alpha_{1}} \wedge \cdots \wedge d x^{\alpha_{p}} \in \Omega_{\mathbb{C}}^{p} \Phi_{\iota}^{q}$. Положим $(x, \omega)=\sum x^{\alpha_{1}} \omega_{\alpha_{1} \ldots \alpha_{p}} d x^{\alpha_{2}} \wedge \cdots \wedge d x^{\alpha_{p}}, \quad\langle\hat{\xi}, \omega\rangle=\sum \widehat{\xi^{\alpha_{1}}} \omega_{\alpha_{1} \ldots \alpha_{p}} d x^{\alpha_{2}} \wedge \cdots \wedge d x^{\alpha_{p}}$,

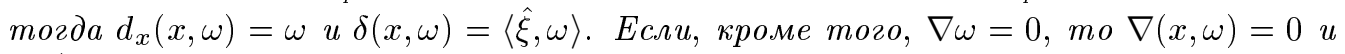
$\nabla\langle\hat{\xi}, \omega\rangle=0$.

ДОКАЗАТЕЛЬСТВО проводится прямыми вычислениями с использованием п. 7 предложения 6.

Лемма 4. Пусть $\phi \in \Phi_{\iota}^{q}$. Равенство $\phi=d_{\mathcal{P}} g$ с некоторым $g \in \mathcal{F}_{\iota}^{q-1}$ имеет место тогда и только тогда, когда

$$
\phi=(\hat{\xi}, \psi)+\nabla \varphi, \quad \psi \in \mathbb{V} \otimes \Phi_{\iota}^{q-1}, \quad \nabla \psi=0, \quad \varphi \in \Phi_{\iota}^{q-1}
$$

(подробнее, $\left.\psi=\left(\psi^{1}, \ldots, \psi^{m}\right), \quad \psi^{\alpha} \in \Phi_{\iota}^{q-1}, \nabla \psi^{\alpha}=0, \quad(\hat{\xi}, \psi)=\sum_{\alpha} \widehat{\xi_{\alpha}} \psi^{\alpha}\right)$.

ДокАЗАТЕЛЬСтво. Пусть $\phi=\phi^{(0)} \in \Phi_{\iota}^{q}, \phi=d_{\mathcal{P}} g$, где $g=g^{(0)} \in \mathcal{F}_{\iota}^{q-1}$. В частности, $d_{x} \phi^{(0)}=0$, откуда $d_{\mathcal{P}} d_{x} g^{(0)}=d_{x} d_{\mathcal{P}} g^{(0)}=0$, так что в силу представления (3.11) имеем $d_{x} g^{(0)}=\phi^{(1)}+d_{\mathcal{P}} g^{(1)}$, где $\phi^{(1)} \in \Omega_{\mathbb{C}}^{1} \Phi_{\iota}^{q-1}, \nabla \phi^{(1)}=0, g^{(1)} \in \Omega^{1} \Phi_{\iota}^{q-2}$. Далее,

$$
d_{\mathcal{P}} d_{x} g^{(1)}=d_{x} d_{\mathcal{P}} g^{(1)}=d_{x}\left(d_{x} g^{(0)}-\phi^{(1)}\right)=0
$$

откуда, опять в силу представления $(3.11)$, имеем $d_{x} g^{(1)}=\phi^{(2)}+d_{\mathcal{P}} g^{(2)}$, где $\phi^{(2)} \in$ $\Omega_{\mathbb{C}}^{2} \Phi_{\iota}^{q-2}, \nabla \phi^{(2)}=0, g^{(2)} \in \Omega^{2} \Phi_{\iota}^{q-3}$, и т.д. На очередном шаге получим $d_{x} g^{(q-1)}=$ $\phi^{(q)}+d_{\mathcal{P}} g^{(q)}$, где $\phi^{(q)} \in \Omega_{\mathbb{C}}^{q} \Phi_{\iota}^{0}, \nabla \phi^{(q)}=0, g^{(q)} \in \Omega^{q} \Phi_{\iota}^{-1}=0$. Итак, $d_{x} g^{(q-1)}=\phi^{(q)}=$ $d_{x}\left(x, \phi^{(q)}\right)$ в силу предложения 7 , так что $d_{x}\left(g^{(q-1)}-\left(x, \phi^{(q)}\right)\right)=0$, и в силу леммы Пуанкаре имеем $g^{(q-1)}=\left(x, \phi^{(q)}\right)+d_{x} h^{(q-2)}$, где $h^{(q-2)} \in \Omega^{q-2} \Phi_{\iota}^{0}$. Далее,

$$
d_{\mathcal{P}} g^{(q-1)}=\delta\left(x, \phi^{(q)}\right)+d_{\mathcal{P}} d_{x} h^{(q-2)}=\left\langle\hat{\xi}, \phi^{(q)}\right\rangle+d_{x} d_{\mathcal{P}} h^{(q-2)}
$$


в силу предложения 7, откуда

$$
\begin{aligned}
d_{x} g^{(q-2)} & =\phi^{(q-1)}+d_{\mathcal{P}} g^{(q-1)}=\phi^{(q-1)}+\left\langle\hat{\xi}, \phi^{(q)}\right\rangle+d_{x} d_{\mathcal{P}} h^{(q-2)}= \\
& =d_{x}\left(\left(x, \tilde{\phi}^{(q-1)}\right)+d_{\mathcal{P}} h^{(q-2)}\right)
\end{aligned}
$$

где $\tilde{\phi}^{(q-1)}=\phi^{(q-1)}+\left\langle\hat{\xi}, \phi^{(q)}\right\rangle \in \Omega_{\mathbb{C}}^{q-1} \Phi_{\iota}^{1}, \nabla \tilde{\phi}^{(q-1)}=0$. Отсюда

$$
g^{(q-2)}=\left(x, \tilde{\phi}^{(q-1)}\right)+d_{\mathcal{P}} h^{(q-2)}+d_{x} h^{(q-3)},
$$

где $h^{(q-3)} \in \Omega^{q-3} \Phi_{\iota}^{1}$, так что

$$
d_{\mathcal{P}} g^{(q-2)}=\delta\left(x, \tilde{\phi}^{(q-1)}\right)+d_{\mathcal{P}} d_{x} h^{(q-3)}=\left\langle\hat{\xi}, \tilde{\phi}^{(q-1)}\right\rangle+d_{x} d_{\mathcal{P}} h^{(q-3)}
$$

и, значит,

$$
d_{x} g^{(q-3)}=\phi^{(q-2)}+\left\langle\hat{\xi}, \tilde{\phi}^{(q-1)}\right\rangle+d_{x} d_{\mathcal{P}} h^{(q-3)}=d_{x}\left(\left(x, \tilde{\phi}^{(q-2)}\right)+d_{\mathcal{P}} h^{(q-3)}\right),
$$

где $\tilde{\phi}^{(q-2)} \in \Omega_{\mathbb{C}}^{q-2} \Phi_{\iota}^{2}, \nabla \tilde{\phi}^{(q-2)}=0$. Продолжая в том же духе, на очередном шаге получим $g^{(2)}=\left(x, \tilde{\phi}^{(3)}\right)+d_{\mathcal{P}} h^{(2)}+d_{x} h^{(1)} \in \Omega^{2} \Phi_{\iota}^{q-3}$, где $h^{(1)} \in \Omega^{1} \Phi_{\iota}^{q-3}, h^{(2)} \in \Omega^{2} \Phi_{\iota}^{q-4}$, $\tilde{\phi}^{(3)} \in \Omega_{\mathbb{C}}^{3} \Phi^{q-3}, \nabla \tilde{\phi}^{(3)}=0$. Далее, $d_{\mathcal{P}} g^{(2)}=\left\langle\hat{\xi}, \tilde{\phi}^{(3)}\right\rangle+d_{x} d_{\mathcal{P}} h^{(1)}$, затем

$$
d_{x} g^{(1)}=\phi^{(2)}+\left\langle\hat{\xi}, \tilde{\phi}^{(3)}\right\rangle+d_{x} d_{\mathcal{P}} h^{(1)}=d_{x}\left(\left(x, \tilde{\phi}^{(2)}\right)+d_{\mathcal{P}} h^{(1)}\right)
$$

откуда $g^{(1)}=\left(x, \tilde{\phi}^{(2)}\right)+d_{\mathcal{P}} h^{(1)}+d_{x} h^{(0)}$, где $\tilde{\phi}^{(2)} \in \Omega_{\mathbb{C}}^{2} \Phi^{q-2}, \nabla \tilde{\phi}^{(2)}=0, h^{(0)} \in \Omega^{0} \Phi_{\iota}^{q-2}=$ $\mathcal{F}_{\iota}^{q-2}$, поэтому $d_{\mathcal{P}} g^{(1)}=\left\langle\hat{\xi}, \tilde{\phi}^{(2)}\right\rangle+d_{x} d_{\mathcal{P}} h^{(0)}$. Таким образом,

$$
d_{x} g^{(0)}=\phi^{(1)}+\left\langle\hat{\xi}, \tilde{\phi}^{(2)}\right\rangle+d_{x} d_{\mathcal{P}} h^{(0)}=d_{x}\left(\left(x, \tilde{\phi}^{(1)}\right)+d_{\mathcal{P}} h^{(0)}\right)
$$

где

$$
\tilde{\phi}^{(1)}=\sum_{\alpha} \tilde{\psi}_{\alpha} d x^{\alpha} \in \Omega_{\mathbb{C}}^{1} \Phi_{\iota}^{q-1}, \quad \nabla \tilde{\psi}_{\alpha}=0, \quad \alpha \in \overline{1, m}
$$

так что $g^{(0)}=\left(x, \tilde{\phi}^{(1)}\right)+d_{\mathcal{P}} h^{(0)}+\varphi, \varphi \in \Omega_{\mathbb{C}}^{0} \Phi_{\iota}^{q-1}=\Phi_{\iota}^{q-1}$ и, значит, $\phi=\sum_{\alpha} \widehat{\xi_{\alpha}} \psi^{\alpha}+\nabla \varphi$, где

$$
\psi^{\alpha}=\sum_{\beta} \chi^{\alpha \beta} \tilde{\psi}_{\beta} \in \Phi_{\iota}^{q-1}, \quad \nabla \psi^{\alpha}=0, \quad \alpha \in \overline{1, m} .
$$

Обратное утверждение очевидно.

Для $p, q=0,1, \ldots$ положим

$$
\Phi_{\iota}^{p, q}=\left\{\phi \in \Phi_{\iota}^{q}: \phi\left(t \xi_{1}, \ldots, t \xi_{q}\right)=t^{p} \phi\left(\xi_{1}, \ldots, \xi_{q}\right) \text { для всех } t>0\right\} .
$$

В частности, $\Phi_{\iota}^{0,0}=\Phi_{\iota}^{0}=\Phi^{0}=\mathbb{C}$, а при $q \geqslant 1$ имеем

$$
\Phi_{\iota}^{q, q}=\left\{\phi\left(\xi_{1}, \ldots, \xi_{q}\right)=\left(\xi_{1} \wedge \cdots \wedge \xi_{q}, c\right) ; c \in \wedge^{q} \mathbb{V}\right\} \simeq \wedge^{q} \mathbb{V}
$$


ПРЕДЛОЖЕНИЕ 8. Пусть $\phi \in \Phi_{\iota}^{q, q}, \quad q \geqslant 1$, тогда $\nabla \phi=0 u \phi=(\hat{\xi}, \psi)$, əде $\psi=$ $\left(\psi^{\alpha}\right) \in \mathbb{V} \otimes \Phi_{\iota}^{q-1, q-1}$.

Первое утверждение проверяется элементарно, а что касается второго, то для

$$
\phi\left(\xi_{1}, \ldots, \xi_{q}\right)=\sum_{\alpha_{1}, \ldots, \alpha_{q}} c^{\alpha_{1} \ldots \alpha_{q}} \xi_{1 \alpha_{1}} \ldots \xi_{q \alpha_{q}}
$$

следует положить

$$
\psi^{\alpha}\left(\xi_{2}, \ldots, \xi_{q}\right)=\sum_{\alpha_{2}, \ldots, \alpha_{q}} c^{\alpha \alpha_{2} \ldots \alpha_{q}} \xi_{2 \alpha_{2}} \ldots \xi_{q \alpha_{q}}
$$

Далее, для $q=0,1, \ldots$ положим

$$
\Phi_{\kappa}^{q}=\left\{\phi \in \Phi_{\iota}^{q}: \phi\left(t \xi_{1}, \ldots, t \xi_{q}\right)=o\left(t^{q}\right) \text { при } t \rightarrow 0\right\} .
$$

По построению $\Phi_{\kappa}^{0}=0$, и легко проверяется, что $\Phi_{\iota}^{q}=\Phi_{\iota}^{q, q} \oplus \Phi_{\kappa}^{q}$, причем $\nabla: \Phi_{\iota}^{q} \rightarrow \Phi_{\kappa}^{q+1}$, для всех $q=0,1, \ldots$ Объединяя эти рассуждения с леммами 3,4 и предложением 8 , убеждаемся, что справедлива

Теорема. Для всех $q=1,2, \ldots$ имеет место представление

$$
H^{q}\left(\left\{\mathcal{F}_{\iota} ; d_{\mathcal{P}}\right\}\right) \simeq \frac{\left\{\phi \in \Phi_{\kappa}^{q}: \nabla \phi=0\right\}}{\left\{\phi=(\hat{\xi}, \psi)+\nabla \varphi ; \psi \in \mathbb{V} \otimes \Phi_{\kappa}^{q-1}, \nabla \psi=0, \varphi \in \Phi_{\kappa}^{q-1}\right\}} .
$$

3.6. Случай $q=0$. По построению $\Phi_{\iota}^{0}=\Phi^{0}=\mathbb{C},\left.\nabla\right|_{\Phi^{0}}=0, \Phi_{\iota}^{-1}=\Phi^{-1}=0$, так что согласно леммам 3 и 4 имеем

$$
H^{0}\left(\left\{\mathcal{F}_{\iota} ; d_{\mathcal{P}}\right\}\right)=\mathbb{C}
$$

В частности, в силу изоморфизмов (1.2) и (3.8)

$$
H^{0}(\mathcal{P} ; \mathcal{P})=\mathbb{C} \text {. }
$$

3.7. Случай $q=1$. Пусть $\phi \in \Phi_{\kappa}^{1}, \nabla \phi=0$. Подробнее, $(\nabla \phi)(\xi, \eta)=-2^{-1}\langle\xi, \eta\rangle \times$ $\nabla_{\xi \eta} \phi=0$ для всех $\xi, \eta \in \mathbb{V}^{*}$. В частности, $\nabla_{\xi \eta} \phi=\phi(\xi+\eta)-\phi(\xi)-\phi(\eta)=0$ для всех $\xi, \eta \in \mathbb{V}^{*}$, откуда с учетом условия $\phi(t \xi)=o(t)$ при $t \rightarrow 0$ следует, что $\phi(\xi)=0$. Итак, в силу теоремы

$$
H^{1}\left(\left\{\mathcal{F}_{\iota} ; d_{\mathcal{P}}\right\}\right)=0 .
$$

Отсюда в силу изоморфизмов (1.2), (3.8) и равенства (3.13) имеем

$$
H^{1}(\mathcal{P} ; \mathcal{P}) \simeq \mathbb{C}
$$

Порождающим коциклом пространства когомологий $H^{1}(\mathcal{P} ; \mathcal{P})$ является, например, отображение $\varepsilon=\Lambda^{-1}[e]: \mathcal{P} \rightarrow \mathcal{P}$, где элемент $e \in \mathcal{F}^{1}$ был определен выше формулой $e(x ; \xi)=1-(\xi, x) / 2$ для всех $x \in V$ и $\xi \in \mathbb{V}^{*}$. Легко проверяется, что

$$
\varepsilon(x ; u)=u(x)-\frac{1}{2} \sum_{\alpha} x^{\alpha} \partial_{x^{\alpha}} u(x)
$$

для всех $x \in V$ и $u(x) \in \mathcal{P}$. 
3.8. Случай $q=2$. Прежде всего заметим, что в силу только что доказанного равенства (3.14) утверждение теоремы в данном случае имеет простой вид:

$$
H^{2}\left(\left\{\mathcal{F}_{\iota} ; d_{\mathcal{P}}\right\}\right) \simeq \frac{\left\{\phi \in \Phi_{\kappa}^{2}: \nabla \phi=0\right\}}{\left\{\phi=\nabla \varphi ; \varphi \in \Phi_{\kappa}^{1}\right\}} .
$$

Пусть $\phi \in \Phi_{\kappa}^{2}, \nabla \phi=0$. В частности, $\phi(0, \eta)=0, \phi(t \xi, t \eta)=o\left(t^{2}\right)$ при $t \rightarrow 0$. Кроме того, в силу п. 3 предложения 6 имеем $\nabla \kappa^{\alpha} \phi=-\kappa^{\alpha} \nabla \phi=0$, причем $\kappa^{\alpha} \phi \in \Phi_{\kappa}^{1}$, так что опять согласно равенству $(3.14) \kappa^{\alpha} \phi=0$, т.е. $\left.\partial_{\xi_{\alpha}} \phi(\xi, \eta)\right|_{\xi=0}=0$ для всех $\alpha \in \overline{1, m}$. Далее, условие $\nabla \phi=0$ имеет вид

$$
\langle\xi, \eta\rangle \nabla_{\xi \eta} \phi(\zeta)+\text { ц.п. }=0 .
$$

В частности, $\langle\xi, \eta\rangle \nabla_{\xi \eta} \phi(\zeta)=0$, а значит, и $\nabla_{\xi \eta} \phi(\zeta)=0$ при $\langle\eta, \zeta\rangle=\langle\zeta, \xi\rangle=0$. Отсюда следует, что

$\nabla_{\xi \eta} \phi(\zeta)=\phi(\xi+\eta, \zeta)-\phi(\xi, \zeta)-\phi(\eta, \zeta)=\langle\xi, \zeta\rangle f(\xi, \eta, \zeta)+\langle\eta, \zeta\rangle g(\xi, \eta, \zeta) \quad \forall \xi, \eta, \zeta \in \mathbb{V}^{*}$

где $f, g$ - некоторые целые функции соответствующего роста. Применяя к этому равенству операцию $\left.\partial_{\xi_{\alpha}}\right|_{\xi=0}, \alpha \in \overline{1, m}$, и учитьвая условие $\left.\partial_{\xi_{\alpha}} \phi(\xi, \eta)\right|_{\xi=0}=0$ и равенство $\partial_{\xi_{\alpha}}\langle\xi, \zeta\rangle=-\zeta^{\alpha}$, получим

$$
\begin{aligned}
\partial_{\eta_{\alpha}} \phi(\eta, \zeta) & =-\zeta^{\alpha} f(0, \eta, \zeta)+\left.\langle\eta, \zeta\rangle \partial_{\xi_{\alpha}} g(\xi, \eta, \zeta)\right|_{\xi=0}= \\
& =\partial_{\eta_{\alpha}}(\langle\eta, \zeta\rangle f(0, \eta, \zeta))+\langle\eta, \zeta\rangle\left(\left.\partial_{\xi_{\alpha}} g(\xi, \eta, \zeta)\right|_{\xi=0}-\partial_{\eta_{\alpha}} f(0, \eta, \zeta)\right)
\end{aligned}
$$

откуда

$$
\partial_{\eta_{\alpha}} \tilde{\phi}(\eta, \zeta)=\langle\eta, \zeta\rangle h^{\alpha}(\eta, \zeta) \quad \forall \alpha \in \overline{1, m}
$$

где

$$
\tilde{\phi}(\eta, \zeta)=\phi(\eta, \zeta)-\langle\eta, \zeta\rangle f(0, \eta, \zeta), \quad h^{\alpha}(\eta, \zeta)=\left.\partial_{\xi_{\alpha}} g(\xi, \eta, \zeta)\right|_{\xi=0}-\partial_{\eta_{\alpha}} f(0, \eta, \zeta)
$$

В частности, $\partial_{\eta_{\alpha}} \tilde{\phi}(\eta, \zeta)=0$ при $\langle\eta, \zeta\rangle=0, \alpha \in \overline{1, m}$, что влечет $\tilde{\phi}(\eta, \zeta)=\nu(\eta)+\langle\eta, \zeta\rangle \times$ $\mu(\eta, \zeta)$, где $\nu(\eta), \mu(\eta, \zeta)$ - некоторые целые функции. Условие $\phi(0, \zeta)=0$ дает $\nu(\eta)=0$, так что

$$
\phi(\eta, \zeta)=\langle\eta, \zeta\rangle \psi(\eta, \zeta)
$$

для всех $\eta, \zeta \in \mathbb{V}^{*}$, где $\psi(\eta, \zeta)=f(0, \eta, \zeta)+\mu(\eta, \zeta)$ - целая функция с необходимыми оценками роста, причем $\psi(\eta, \zeta)=\psi(\zeta, \eta)$, поскольку $\phi(\eta, \zeta)=-\phi(\zeta, \eta)$ и, кроме того, $\psi(0, \zeta)=0$ в силу условия $\left.\partial_{\xi_{\alpha}} \phi(\xi, \zeta)\right|_{\xi=0}=0$.

Далее,

$$
\begin{aligned}
\nabla_{\xi \eta} \phi(\zeta) & =\langle\xi+\eta, \zeta\rangle \psi(\xi+\eta, \zeta)-\langle\xi, \zeta\rangle \psi(\xi, \zeta)-\langle\eta, \zeta\rangle \psi(\eta, \zeta)= \\
& =\langle\xi, \zeta\rangle \Delta_{\eta} \psi(\xi, \zeta)+\langle\eta, \zeta\rangle \Delta_{\xi} \psi(\eta, \zeta),
\end{aligned}
$$


где, например, $\Delta_{\eta} \psi(\xi, \zeta)=\psi(\xi+\eta, \zeta)-\psi(\xi, \zeta)$. Подставляя это и аналогичные выражения, получаемые циклическими перестановками, в равенство (3.15), получим

$$
\langle\zeta, \xi\rangle\langle\xi, \eta\rangle \Delta \psi(\xi, \eta, \zeta)+\text { ц.п. }=0,
$$

где $\Delta \psi(\xi, \eta, \zeta)=\Delta_{\zeta} \psi(\xi, \eta)-\Delta_{\eta} \psi(\xi, \zeta)$. Легко проверяется тождество

$$
\Delta \psi(\xi, \eta, \zeta)+\text { ц.п. }=0
$$

для всех функций $\psi(\xi, \eta)$ и аргументов $\xi, \eta, \zeta \in \mathbb{V}^{*}$. В частности,

$$
\Delta \psi(\zeta, \xi, \eta)=-\Delta \psi(\xi, \eta, \zeta)-\Delta \psi(\eta, \zeta, \xi) .
$$

Подстановка этого выражения в (3.17) дает

$$
\langle\xi, \zeta\rangle\langle\xi+\zeta, \eta\rangle \Delta \psi(\xi, \eta, \zeta)+\langle\eta, \zeta\rangle\langle\eta+\zeta, \xi\rangle \Delta \psi(\eta, \zeta, \xi)=0,
$$

откуда

$$
\begin{aligned}
\langle\xi, \zeta\rangle\langle\xi+\zeta, \eta\rangle \Delta \psi(\xi, \eta, \zeta) & =-\langle\eta, \zeta\rangle\langle\eta+\zeta, \xi\rangle \Delta \psi(\eta, \zeta, \xi)= \\
& =\langle\xi, \zeta\rangle\langle\xi+\zeta, \eta\rangle\langle\eta, \zeta\rangle\langle\eta+\zeta, \xi\rangle g(\xi, \eta, \zeta),
\end{aligned}
$$

где $g(\xi, \eta, \zeta)$ - некоторая целая симметрическая функция аргументов $\xi, \eta, \zeta \in \mathbb{V}^{*}$. В частности,

$$
\Delta \psi(\xi, \eta, \zeta)=\langle\eta, \zeta\rangle\langle\eta+\zeta, \xi\rangle g(\xi, \eta, \zeta)
$$

Применив к этому равенству операцию $\left.\partial_{\eta_{\alpha}}\right|_{\eta=0}, \alpha \in \overline{1, m}$, получим

$$
\partial_{\xi_{\alpha}} \psi(\xi, \zeta)=\rho^{\alpha}(\xi+\zeta)-\rho^{\alpha}(\xi)-\zeta^{\alpha}\langle\xi, \zeta\rangle h(\xi, \zeta),
$$

где $\rho^{\alpha}(\xi)=\left.\partial_{\eta_{\alpha}} \psi(\xi, \eta)\right|_{\eta=0}, h(\xi, \zeta)=g(\xi, 0, \zeta)=h(\zeta, \xi)$. Равенство смешанных производных приводит к условиям разрешимости

$$
\begin{aligned}
\partial_{\xi_{\alpha}} \partial_{\xi_{\beta}} \phi(\xi, \zeta) & =\partial_{\xi_{\alpha}}\left(\rho^{\beta}(\xi+\zeta)-\rho^{\beta}(\xi)\right)-\langle\xi, \zeta\rangle \zeta^{\beta} \partial_{\xi_{\alpha}} h(\xi, \zeta)= \\
& =\partial_{\xi_{\beta}}\left(\rho^{\alpha}(\xi+\zeta)-\rho^{\alpha}(\xi)\right)-\langle\xi, \zeta\rangle \zeta^{\alpha} \partial_{\xi_{\beta}} h(\xi, \zeta)
\end{aligned}
$$

для всех $\alpha, \beta \in \overline{1, m}$. Другими словами,

$$
\begin{aligned}
\partial_{\xi_{\alpha}} & \left(\rho^{\beta}(\xi+\zeta)-\rho^{\beta}(\xi)\right)-\partial_{\xi_{\beta}}\left(\rho^{\alpha}(\xi+\zeta)-\rho^{\alpha}(\xi)\right)= \\
& =\langle\xi, \zeta\rangle\left(\zeta^{\beta} \partial_{\xi_{\alpha}}-\zeta^{\alpha} \partial_{\xi_{\beta}}\right) h(\xi, \zeta) .
\end{aligned}
$$

Полагая здесь $\xi=0$, получим

$$
\partial_{\zeta_{\alpha}} \rho^{\beta}(\zeta)-\partial_{\zeta_{\beta}} \rho^{\alpha}(\zeta)=\left.\partial_{\xi_{\alpha}} \rho^{\beta}(\xi)\right|_{\xi=0}-\left.\partial_{\xi_{\beta}} \rho^{\alpha}(\xi)\right|_{\xi=0}=0,
$$


поскольку

$$
\left.\partial_{\xi_{\alpha}} \rho^{\beta}(\xi)\right|_{\xi=0}=\left.\partial_{\xi_{\alpha}} \partial_{\eta_{\beta}} \psi(\xi, \eta)\right|_{\xi=\eta=0}=\left.\partial_{\xi_{\beta}} \rho^{\alpha}(\xi)\right|_{\xi=0}
$$

согласно условию $\psi(\xi, \eta)=\psi(\eta, \xi)$. Следовательно, в силу леммы Пуанкаре существует функция $\rho(\xi) \in \Phi^{1}, \rho(0)=0$, такая, что $\rho^{\alpha}(\xi)=\partial_{\xi_{\alpha}} \rho(\xi)$. Условия разрешимости (3.19) теперь принимают вид

$$
\left(\zeta^{\beta} \partial_{\xi_{\alpha}}-\zeta^{\alpha} \partial_{\xi_{\beta}}\right) h(\xi, \zeta)=0 \quad \forall \alpha, \beta \in \overline{1, m}
$$

и имеют обшее решение $h(\xi, \zeta)=k(\langle\xi, \zeta\rangle)$, где $k(z)$ - целая функция аргумента $z \in \mathbb{C}$. Равенство (3.18) можно переписать теперь в виде

$$
\partial_{\xi_{\alpha}}\left(\psi(\xi, \zeta)-\nabla_{\xi \zeta} \rho-K(\langle\xi, \zeta\rangle)\right)=0 \quad \forall \alpha \in \overline{1, m}
$$

где $K(z)$ - целая функция такая, что $-\zeta^{\alpha}\langle\xi, \zeta\rangle k(\langle\xi, \zeta\rangle)=\partial_{\xi_{\alpha}} K(\langle\xi, \zeta\rangle), K(0)=0$. Итак,

$$
\psi(\xi, \zeta)=\nabla_{\xi \zeta} \rho+K(\langle\xi, \zeta\rangle)+c(\zeta)
$$

где $c(\zeta)$ - некоторая функция. Учитьвая, что $\psi(0, \zeta)=0, \rho(0)=0, K(0)=0$, находим $c(\zeta)=0$. Условие $\psi(\xi, \zeta)=\psi(\zeta, \xi)$ дает $K(z)=K(-z)$, так что $K(z)=F\left(z^{2}\right)$, где $F(z)$ - другая целая функция, $F(0)=0$. Таким образом,

$$
\psi(\xi, \zeta)=\nabla_{\xi \zeta} \rho+F\left(\langle\xi, \zeta\rangle^{2}\right) .
$$

Здесь $\rho(\xi) \in \Phi^{1}, \rho(0)=0$, более того, можно считать, что $\left.\partial_{\xi_{\alpha}} \rho(0)\right|_{\xi=0}=0$ для всех $\alpha \in \overline{1, m}$, поскольку $\nabla_{\xi \zeta} \tilde{\rho}=0$ для любой линейной функции $\tilde{\rho}(\xi)=(\xi, c)=\sum_{\alpha} c^{\alpha} \xi_{\alpha}$, $c \in \mathbb{V}$. Итак, с учетом равенства (3.16) получаем представление

$$
\phi=\tilde{\phi}+\nabla \varphi
$$

где $\varphi(\xi)=-2 \rho \in \Phi_{\kappa}^{1}, \tilde{\phi}(\xi, \eta)=\langle\xi, \eta\rangle \tilde{\psi}(\xi, \eta), \tilde{\psi}(\xi, \eta)=F\left(\langle\xi, \eta\rangle^{2}\right), F(z)$ - целая функция соответствующего роста, $F(0)=0$.

Лемма 5. Для данной целой функиии $\psi(\xi, \eta)=\psi(\eta, \xi), \psi(0, \eta)=0$, существует челая функиия $\rho(\xi), \quad \rho(0)=0$, такая, что $\psi(\xi, \eta)=\nabla_{\xi \eta} \rho$, тогда и только тогда, когда $\Delta \psi=0$.

ДоКАЗАТЕЛЬСТВо. Напомним, что

$$
\nabla_{\xi \eta} \rho=\rho(\xi+\eta)-\rho(\xi)-\rho(\eta), \quad \Delta \psi(\xi, \eta, \zeta)=\psi(\xi+\zeta, \eta)-\psi(\xi, \eta)-\psi(\xi+\eta, \zeta)+\psi(\xi, \zeta) .
$$

Легко проверяется, что представление $\psi(\xi, \eta)=\nabla_{\xi \eta} \rho$ влечет равенство $\Delta \psi=0$. Итак, предположим, что $\Delta \psi=0$ для данной функции $\psi$ с указанными свойствами. Применяя к этому равенству операцию $\left.\partial_{\zeta_{\alpha}}\right|_{\zeta=0}, \alpha \in \overline{1, m}$, получим соотношения

$$
\partial_{\xi_{\alpha}} \psi(\xi, \eta)-\rho^{\alpha}(\xi+\eta)+\rho^{\alpha}(\xi)=0, \quad \rho^{\alpha}(\xi)=\left.\partial_{\zeta_{\alpha}} \psi(\xi, \zeta)\right|_{\zeta=0} .
$$


Равенство смешанных производных приводит к условиям разрешимости

$$
\partial_{\xi_{\alpha}} \partial_{\xi_{\beta}} \psi(\xi, \eta)=\partial_{\xi_{\alpha}}\left(\rho^{\beta}(\xi+\eta)-\rho^{\beta}(\xi)\right)=\partial_{\xi_{\beta}}\left(\rho^{\alpha}(\xi+\eta)-\rho^{\alpha}(\xi)\right)
$$

для всех $\alpha, \beta \in \overline{1, m}$. Положив $\xi=0$, получим

$$
\partial_{\eta_{\alpha}} \rho^{\beta}(\eta)-\partial_{\eta_{\beta}} \rho^{\alpha}(\eta)=\left.\partial_{\xi_{\alpha}} \rho^{\beta}(\xi)\right|_{\xi=0}-\left.\partial_{\xi_{\beta}} \rho^{\alpha}(\xi)\right|_{\xi=0}=0
$$

поскольку $\left.\partial_{\xi_{\alpha}} \rho^{\beta}(\xi)\right|_{\xi=0}=\left.\partial_{\xi_{\alpha}} \partial_{\zeta_{\beta}} \psi(\xi, \zeta)\right|_{\xi=\zeta=0}=\left.\partial_{\xi_{\beta}} \rho^{\alpha}(\xi)\right|_{\xi=0}$, так как по условию леммы $\psi(\xi, \eta)=\psi(\eta, \xi)$. Отсюда в силу леммы Пуанкаре найдется целая функция $\rho(\eta)$, $\rho(0)=0$, такая, что $\rho^{\alpha}(\eta)=\partial_{\eta_{\alpha}} \rho(\eta), \alpha \in \overline{1, m}$. Подставляя это представление в $(3.21)$, получим

$$
\partial_{\xi_{\alpha}}(\psi(\xi, \eta)-\rho(\xi+\eta)+\rho(\xi))=0 \quad \forall \alpha \in \overline{1, m},
$$

так что

$$
\psi(\xi, \eta)=\rho(\xi+\eta)-\rho(\xi)+c(\eta),
$$

а подстановка $\psi(0, \eta)=0, \rho(0)=0$ дает $c(\eta)=-\rho(\eta)$, что и требовалось доказать.

Итак, для того чтобы найти нетривиальные 2-коциклы, следует среди целых функций вида $\psi(\xi, \eta)=F\left(\langle\xi, \eta\rangle^{2}\right)$ выделить такие, что $\Delta \psi \neq 0$, но выполнено равенство (3.17). Учитывая, что операция $\Delta$ переводит однородный многочлен в однородный, достаточно провести указанную проверку для однородных многочленов вида $\psi(\xi, \eta)=\langle\xi, \eta\rangle^{2 n}, n=$ $1,2, \ldots$. Элементарные вычисления показывают, что в этом случае $\Delta \psi \neq 0$ для всех $n=1,2, \ldots$, в то время как равенство (3.17) вьполнено лишь для $n=1$. Таким образом,

$$
H^{2}\left(\left\{\mathcal{F}_{\iota} ; d_{\mathcal{P}}\right\}\right)=\mathbb{C}
$$

причем порождаюшим коциклом является элемент $s(\xi, \eta)=\langle\xi, \eta\rangle^{3} \in \mathcal{F}_{\iota}^{2}$. Отсюда в силу изоморфизмов $(1.2),(3.8)$ и равенства (3.14) имеем

$$
H^{2}(\mathcal{P} ; \mathcal{P}) \simeq \mathbb{C}
$$

причем в качестве порождающего коцикла здесь можно взять 2 -коцепь $\sigma=\Lambda^{-1}[s]: \mathcal{P} \times$ $\mathcal{P} \rightarrow \mathcal{P}$, где

$$
\sigma(x ; u, v)=\left.\left\langle\partial_{y}, \partial_{z}\right\rangle^{3} u(y) v(z)\right|_{y=z=x} \quad \forall u, v \in \mathcal{P} .
$$

3.9. Обсуждение. Приведенная выше теорема кардинально упрошает вычисление когомологий алгебры Пуассона с коэффициентами в присоединенном представлении.

Равенство $H^{0}(\mathcal{P} ; \mathcal{P})=\mathbb{C}$ означает, что центр алгебры Пуассона

$$
\text { cen } \mathcal{P}=\{u \in \mathcal{P}:\{u, v\}=0 \text { для всех } v \in \mathcal{P}\}=\mathbb{C} .
$$

Изоморфизм $H^{1}(\mathcal{P} ; \mathcal{P}) \simeq \mathbb{C}$ означает, что алгебра Пуассона имеет единственное нетривиальное внешнее дифферениирование, или, другими словами, единственное нетривиальное одномерное правое расширение. 
Изоморфизм $H^{2}(\mathcal{P} ; \mathcal{P}) \simeq \mathbb{C}$ означает, что алгебра Пуассона имеет единственную нетривиальную инфинитезимальную деформацию. Заметим, что в книге [8] и обзоpe [9] для размерности $m=2$ приведен 2 -коцикл $\omega: \mathcal{P} \times \mathcal{P} \rightarrow \mathcal{P}$, где

$$
\omega(x ; u, v)=\left(\partial_{x^{1}} \partial_{x^{1}} u(x)\right)\left(\partial_{x^{2}} \partial_{x^{2}} v(x)\right)-\left(\partial_{x^{2}} \partial_{x^{2}} u(x)\right)\left(\partial_{x^{1}} \partial_{x^{1}} v(x)\right)
$$

для всех $x=\left(x^{1}, x^{2}\right) \in \mathbb{R}^{2}$ и $u, v \in \mathcal{P}$, и утверждается, что он порождает пространство $H^{2}(\mathcal{P} ; \mathcal{P}) \simeq \mathbb{C}$. В действительности этот коцикл тривиальный, а именно $\omega=d_{\mathcal{P}} \rho$, где 1 -коцикл $\rho: \mathcal{P} \rightarrow \mathcal{P}$ задается правилом

$$
\rho(x ; u)=-2 \partial_{x^{1}} \partial_{x^{2}} u(x)
$$

для всех $x \in \mathbb{R}^{2}$ и $u \in \mathcal{P}$.

Благодарности. Работа вьполнена при поддержке гранта РФФИ № 01-01-00516.

\section{Список литературы}

[1] N. Kamran, P. J. Olver (Eds.). Lie algebras, Cohomology, and New Applications to Quantum Mechanics. Contemp. Math. V. 160. Providence, Rhode Island: AMS, 1994.

[2] J.A. de Azcárraga, J. M. Izquierdo, J. C. Pérez Bueno. An introduction to some novel applications of Lie algebra cohomology in mathematics and physics. physics/9803046.

[3] M. Henneaux, J. Krasil'shchik, A. Vinogradov (Eds.). Secondary Calculus and Cohomological Physics. Contemp. Math. V. 219. Providence, Rhode Island: AMS, 1998.

[4] M. Bordemann. The deformation quantization of certain super-Poisson brackets and BRST cohomology. math.QA/0003218; P. Monnier. Poisson cohomology in dimension two. math.DG/0005261; Computation of Nambu-Poisson cohomologies. math.DG/0007103; Z. Giunashvili. Differential complex of Poisson manifold and distributions. math-ph/0103038; Noncommutative geometry of phase space. math-ph/0203027; O. Radko. A classification of topologicaly stable Poisson structures on a compact oriented surface. math.SG/0110304; $V$. V. Kornyak. Computation of cohomology of Lie algebra of Hamiltonian vector fields by splitting cochain complex into minimal subcomplexes. math.NA/0205046; A. Gammella. Pacific. J. Math. 2002. V. 203. P. 280-320; math.DG/0207215; J. A. de Azcárraga, J. M. Izquierdo, J. C. Pérez Bueno. J. Phys. A. 1997. V. 30. P. L607-L616; hep-th/9703019; S. Evens, J.-H. Lu. Poisson harmonic forms, Kostant harmonic forms, and the $S^{1}$-equivariant cohomology of $K / T$. dg-ga/9711019; M. Penkava, P. Vanhaecke. Deformation quantization of polynomial Poisson algebras. math.QA/9804022.

[5] В. В. Жаринов. ТМФ. 2001. Т. 128. № 2. С. 147-160.

[6] Н. Бурбаки. Группы и алгебры Ли. М.: Мир, 1976.

[7] П. Картье. Когомологии алгебр Ли. В сб.: Теория алгебр Ли. Топология групп Ли. Семинар "Софус Ли". Ред. Е. Б. Дынкин. М.: ИЛ, 1962. С. 32.

[8] Д. Б. Фукс. Когомологии бесконечномерных алгебр Ли. М.: Наука, 1984.

[9] Б. Л. Фейгин, Д. Б. Фукс. Когомологии групп и алгебр Ли. В сб.: Итоги науки и техники. Современные проблемы математики. Фундаментальные направления. Т. 21. Ред. Р. В. Гамкрелидзе. М.: ВИНИТИ, 1988. С. 121.

[10] Л. Хермандер. Анализ линейных дифференциальных операторов с частными производными. Т. 1. Теория распределений и анализ Фурье. М.: Мир, 1986.

Поступила в редакцию 15.XII.2002 г. 